\title{
Colección de Mamíferos, Museo de Ciencias Naturales de La Salle del Instituto Tecnológico Metropolitano (CSJ-m)
}

\author{
Danny Zurc ${ }^{1 *}$ (D) Andrea Bustca1 ${ }^{(\mathbb{D})}$, Estefanía Arbeláez-Rendón ${ }^{1}$ (D) \\ Oscar S. Alzate-Zapata² \\ 1 Museo de Ciencias Naturales de La Salle, Instituto Tecnológico Metropolitano. Calle 54A \# 30-01, bloque L, oficina 307, \\ Medellín, Antioquia. \\ 2 Universidad Eafit, Programa de Biología. Carrera 49 \# 7 sur - 50, Medellín, Antioquia. \\ *Correspondencia: dannyzurc@gmail.com
}

\section{Resumen}

La Colección de Mamíferos del Museo de Ciencias Naturales de La Salle (CSJ-m), alberga 1226 especímenes de mamíferos pertenecientes a 12 órdenes, 37 familias, 89 géneros y 146 especies (seis exóticas). Los especímenes proceden de 19 departamentos del país y cinco países extranjeros. La colección es la más antigua del departamento de Antioquia y la segunda más antigua de Colombia, después del Museo de La Salle, Bogotá.

Palabras clave: Antioquia, colecciones biológicas, ejemplares zoológicos, mastozoología, patrimonio natural.

\begin{abstract}
The Mammals Collection of the Museo de Ciencias Naturales de La Salle (CSJ-m), holds 1226 specimens belonging to 12 orders, 37 families, 89 genera, and 146 species (six exotic). The specimens come from 19 departments in the country and five countries overseas. The collection is the oldest of the Department of Antioquia and the second oldest of Colombia, after the Museo de La Salle, Bogotá.
\end{abstract}

Key words: Antioquia, biological collections, zoological specimens, mammalogy, natural heritage.

La Colección de Mamíferos del Museo de Ciencias Naturales de La Salle (CSJ-m) (Figura 1), se ubica en la ciudad de Medellín, en el Instituto Tecnológico Metropolitano (ITM) y fue fundada en 1913 en el Colegio San José (Medellín) por iniciativa del Hermano Nicéforo María, de las Escuelas Cristianas Lasallistas. Durante 100 años fue enriquecida por diferentes colectores, entre ellos se destacan el Hermano Daniel González Patiño, Hno. Marco Antonio Serna, Hno. José Ignacio Isaza, Hno. Octavio Martínez, Hno. Luis Alberto Zamudio, el Club Científico Colombiano (3C), Horacio Echeverri, Rafael Borja, Afranio Ortiz, entre otros. En el año 2004 el Museo cierra sus puertas debido a negociaciones administrativas de la institución que en ese entonces custodiaba dicho centro museal. En 
el año 2011 nuevamente se da apertura a este lugar, y es a partir del año 2013 que diversos colectores empiezan a depositar especímenes para su custodia, aumentando en más del 50 \% el número de individuos de la colección.

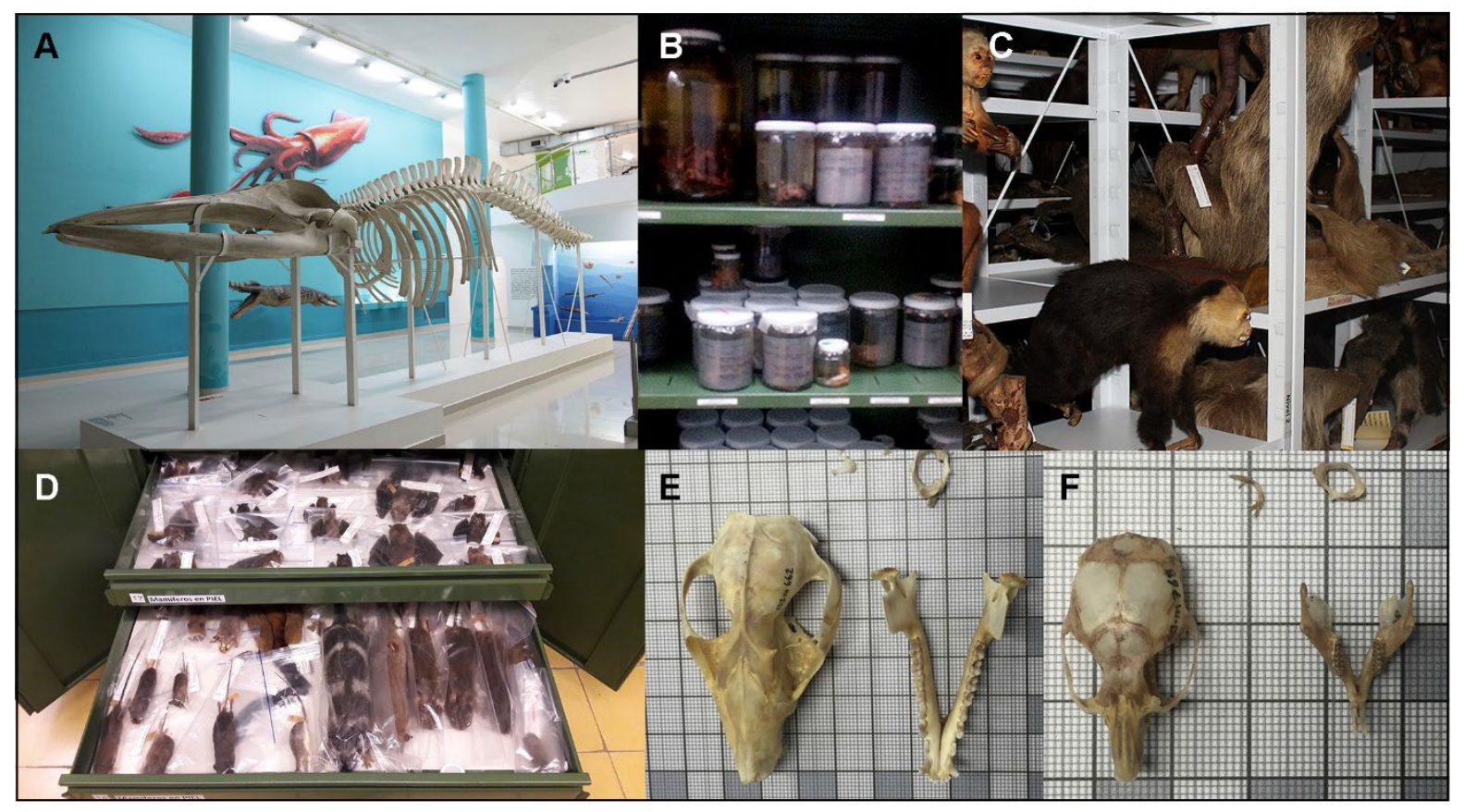

FIGURA 1. Colección de Mamíferos (CSI-m) Museo de Ciencias Naturales de La Salle. A, Sala de exposición. B, Detalle de la colección en líquido. C, Detalle de la colección en taxidermia para exposición. D, Detalle de la colección de pieles para estudio. E - F, Detalle de los cráneos de las pieles para estudio.

En la actualidad, la colección alberga 1226 ejemplares catalogados, pertenecientes a 12 órdenes, 37 familias, 89 géneros y 146 especies (seis exóticas: Chlamyphorus truncatus, Condylura cristala, Erinaceaus europaeus, Jaculus jaculus, Ondatra zibethicus, Tachyorictes splendens) (Tabla 1). Los órdenes mejor representados son Chiroptera y Rodentia con 80 y 22 especies respectivamente. Un total de 24 especímenes no han sido identificados a nivel de género. Los especímenes proceden de los departamentos de Antioquia (945), Atlántico (33), Bolívar (18), Caldas (16), Caquetá (2), Casanare (2), Cesar (2), Chocó (4), Córdoba (33), Cundinamarca (5), La Guajira (30), Magdalena (49), Meta (1), Nariño (5), Norte de Santander (42), Risaralda (7), Santander (7), Tolima (2), Valle del Cauca (14), y de los países Argentina, Bélgica, Estados Unidos, Sudán y Uganda (9).

Especímenes de la colección de mamíferos del Museo (CSJ-m), han sido empleados en al menos 4 publicaciones científicas (Arias-Alzate et al. 2010; Martínez-Arias et al. 2010; Martínez-Arias \& Solari 2013; Martínez-Cerón et al. 2019).

En la actualidad el Museo se encuentra desarrollando diferentes estrategias de carácter divulgativo, buscando la captación de público especializado para la consulta de las colecciones, como las investigaciones con especímenes de mamíferos. Finalmente, este centro científico apuesta a la generación de espacios para la apropiación del conocimiento a partir de proyectos expositivos, trabajos con estudiantes del ITM, Universidad Eafit y Universidad de Antioquia, junto con comunidades vecinas, a fin de comunicar la importancia del patrimonio natural que alberga. 
Mamíferos del Museo de Ciencias Naturales de La Salle.

\begin{tabular}{lcccc}
\hline \multicolumn{1}{c}{ Orden } & Familias & Géneros & Especies & Número de especímenes \\
\hline Didelphimorphia & 1 & 7 & 11 & 35 \\
Paucituberculata & - & - & - & - \\
Cingulata & 1 & 1 & 1 & 12 \\
Pilosa & 4 & 4 & 4 & 13 \\
Sirenia & - & - & - & - \\
Eulipotyphla & 1 & 1 & 1 & 4 \\
Chiroptera & 8 & 35 & 80 & 736 \\
Carnivora & 4 & 10 & 10 & 28 \\
Perissodactyla & 1 & 1 & 1 & 3 \\
Artiodactyla & 2 & 3 & 4 & 10 \\
Cetacea & 1 & 1 & 3 & 6 \\
Primates & 5 & 7 & 8 & 14 \\
Rodentia & 8 & 18 & 22 & 363 \\
Lagomorpha & 1 & 1 & 1 & 2 \\
Total & 37 & 89 & 146 & 1226 \\
\hline
\end{tabular}

\section{AGRADECIMIENTOS}

A Frank Vélez Penagos del Área de Arte y Diseño del Museo de Ciencias Naturales de La Salle por su apoyo en la realización de fotografias de la colección.

\section{REFERENCIAS}

Arias-Alzate A, Downer CC, Delgado-V CA, Sánchez-Londoño JD. 2010. Un registro de tapir de montaña (Tapirus pinchaque) en el norte de la cordillera Occidental de Colombia. Mastozoología Neotropical 17:111-116.

Martínez-Arias A, Solari S, Díaz-Nieto JF. 2010. Re-evaluation of a Colombian record of Sturnira thomasi De La Torre \& Schwartz, 1966 (Phyllostomidae: Stenodermatinae). Chiroptera Neotropical 16:603-609.

Martínez-Arias VM, Solari S. 2013. Extensión del área de distribución de Sturnira koopmanhilli McCarthy et al. 2006 en Colombia. Therya 4:617-625. http://dx.doi.org/10.12933/therya-13152

Martínez-Cerón JM, Patiño-Castillo E, Carvalho-Madrigal S, Díaz-Nieto JF. 2019. Molecular and morphological identification of Phylloderma stenops Peters, 1865 (Chiroptera, Phyllostomidae) and new records for Colombia. Check List 15:37-44. https:/ / doi.org/10.15560/15.1.37

Editor: Miguel E. Rodríguez-Posada Recibido 2020-04-14 Revisado 2020-04-17 Aceptado 2020-04-27 Publicado 2020-07-01 